\title{
Crystal growth and Characterization of an NLO material: L-cysteinium Sodium Nitrate Chloride (LCSNC)
}

\author{
V. Azeezaa, ${ }^{1}$ A. Joseph Arul Pragasam, ${ }^{* 2}$ T.G. Sunitha ${ }^{3}$ \\ ${ }^{1}$ Research Scholar, Sathyabama University, Chennai- 603 103, India, \\ ${ }^{2}$ Faculty of Physics, Sathyabama University, Chennai- 600 119, India, \\ ${ }^{3}$ Faculty of Chemistry, Pachaiyappa's college, Chennai- 600 030, India
}

Received July 10, 2014; accepted September 16, 2014; published September 30, 2014

\begin{abstract}
In the present work, L-Cysteinium Sodium Nitrate Chloride (LCSNC) an organic nonlinear optical material has been synthesized by a slow evaporation method. The crystalline nature was confirmed by single crystals $\mathrm{X}$-ray diffraction analysis. The UV-Vis transmittance spectrum shows that the crystal has a good optical transmittance in the entire visible region with lower cutoff wavelength $203 \mathrm{~nm}$. The various functional groups and chemical compositions of the grown crystal have been derived from FTIR spectral analysis. Both dielectric constant and dielectric loss decrease with an increase in frequency. The SHG efficiency was measured by employing the powder Kurtz method.
\end{abstract}

The search for new materials with high optical nonlinearity is an important area due to their applications like optical communication, optical computing, optical information processing, optical disk data storage, laser fusion reactions, laser remote sensing, colour display, medical diagnostics, and so forth [1-2]. Most of the organic NLO crystals usually have poor mechanical and thermal properties and are susceptible to damage during processing even though they have large NLO efficiency. Purely inorganic NLO materials have excellent mechanical and thermal properties but possess relatively modest optical non linearity because of the lack of extended pi-electron delocalization. Organic NLO materials are often superior to inorganic in terms of their response speed, optical clarity, and the magnitude of their third order susceptibility. However, for a material to exhibit NLO activity it should be noncentrosymmetric. Also organic materials have a high nonlinear optical coefficient, higher laser damage threshold, fast response, low mobility, and their large band gap finds many applications [3-4].

L-cysteine is an organic compound under an amino acid category. It is one of the naturally occurring protenogenic amino acids. L-cysteine exists in a zwitterionic form; the molecule can combine with anionic, cationic and overall neutral constituents. In this paper we report the growth, structural, vibrational, optical, dielectric, and second harmonic generation (SHG)

\footnotetext{
*E-mail: jap.azee.phy@gmail.com
}

properties of single crystals of L-cysteinium sodium nitrate chloride (LCSNC).

The material was synthesized by taking L-cysteine and sodium nitrate in a 1:1 stoichiometric ratio.

A calculated amount of these materials was taken and dissolved in deionized water then hydrochloric acid was added drop by drop to reduce $\mathrm{pH}$ less than 2 , then the solution was filtered by a Whatmann filter paper of the $11 \mu \mathrm{m}$ pore size [5]. The prepared solution was allowed to dry at room temperature and the salts were obtained by a slow evaporation technique. The purity of the synthesized salt was further improved by a successive recrystallization process yielding a crystal of the size $21 \times 7 \times 9 \mathrm{~mm}^{3}$ in a growth period of 27 days. The grown single crystal of LCSNC is shown in Fig. 1.

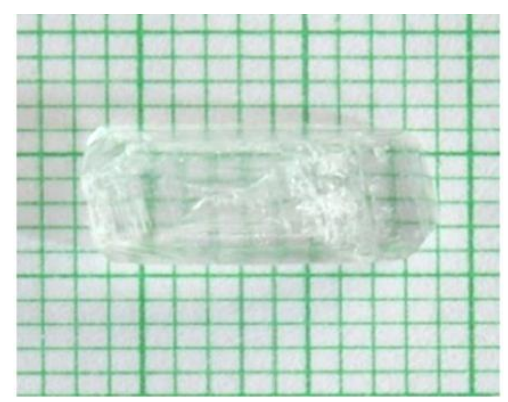

Fig. 1. The grown LCSNC crystal.

The single crystal X-ray diffraction analysis of the crystal indicates that it crystallizes in a monoclinic system with noncentrosymmetric space group $\mathrm{P} 2{ }_{1}$ with lattice parameters: $\mathrm{a}=18.5 \AA, \quad \mathrm{b}=5.22 \AA, \quad \mathrm{c}=7.22 \AA, \quad \alpha=90^{\circ}$, $\beta=103.83^{\circ}, \gamma=90^{\circ}$ and $V=678 \AA^{3}$.

The UV-Vis spectrum gives information about the structure of the molecule that the absorption of UV and visible light involves in the promotion of electron in $\sigma$ and $\pi$ orbital from the ground state to the higher energy state. The transmittance spectrum was recorded with a Varian Carry-5E UV-Vis spectrophotometer in the wavelength range 200-1400nm. The UV transmission spectrum of the 
grown crystal is shown in Fig. 2. The determination of UV transparency and cutoff wavelength is very important since these crystals are mainly used in optical application. The lower cutoff wavelength is found to be $203 \mathrm{~nm}$ and the upper cutoff wavelength is $328 \mathrm{~nm}$. Between $347 \mathrm{~nm}$ and $1400 \mathrm{~nm}$, there is no absorption of wavelength which clearly indicates that grown crystals can be used as a window material in optical instruments. The small peak at $241 \mathrm{~nm}$ is due to $\mathrm{n}-\pi^{*}$ transition [6]. High transmittance observed from $347 \mathrm{~nm}$ indicates that the crystal possesses good optical transparency for SHG of Nd:YAG laser.

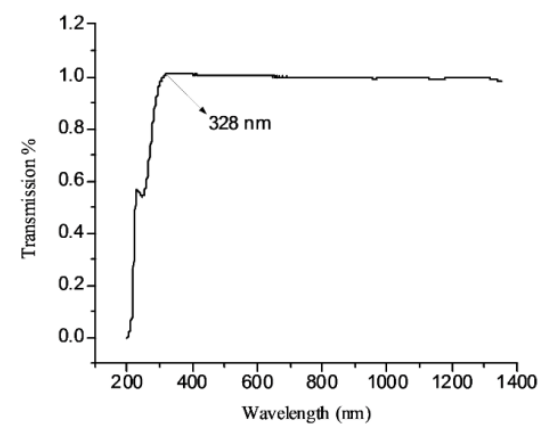

Fig. 2. Transmission spectrum of LCSNC crystal.

A Fourier transform infrared spectrum was recorded using a Bruker IFS 66V FTIR spectrometer in the range of $600-4000 \mathrm{~cm}^{-1}$ and the recorded FTIR spectrum is shown in Fig. 3. Vibrational spectroscopy provides an important tool to understand chemical bonding.

The strong absorption peak at $3421 \mathrm{~cm}^{-1}$ is due to O$\mathrm{H}$ symmetric stretching [7]. The frequencies observed at 2970 and $2905 \mathrm{~cm}^{-1}$ are attributed to $\mathrm{NH}_{3}{ }^{+}$stretching and $\mathrm{C}-\mathrm{H}$ stretching. The absorption peaks observed at 1130 and $1048 \mathrm{~cm}^{-1}$ respond to the $\mathrm{C}-\mathrm{N}$ group. The peaks observed at 813,746 and $608 \mathrm{~cm}^{-1}$ is attributed to the $\mathrm{CH}_{2}$ group while the peak observed at $1635 \mathrm{~cm}^{-1}$ is attributed to the $\mathrm{COO}^{-}$group. The bands observed at 1593 and $1381 \mathrm{~cm}^{-1}$ are attributed to $\mathrm{CO}_{2}$ symmetric and asymmetric stretching. The presence of these bands showed that the adsorbed L-cysteine was to be in its Zwitterionic state.

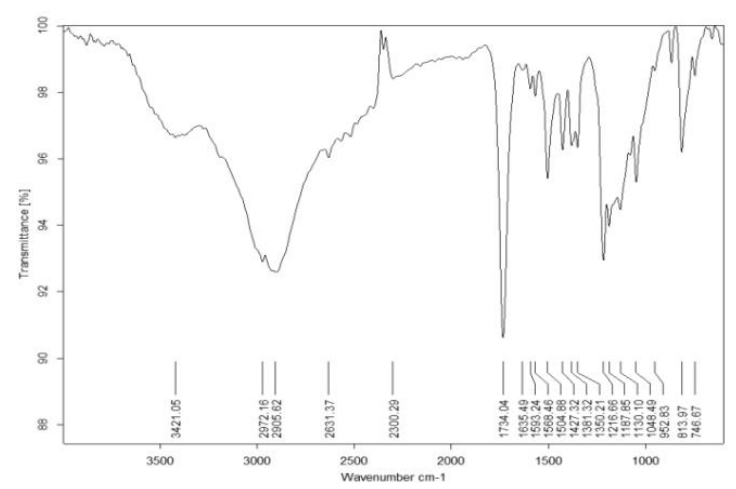

Fig. 3. FTIR spectrum of the grown crystal LCSNC.
The dielectric properties are associated with the electro-optic property of materials, particularly when they are non-conducting materials [8]. Microelectronic industry needs low $\varepsilon_{\mathrm{r}}$ materials as an interlayer dielectric. Figures 4 and 5 show the variation of dielectric constant and dielectric loss of the title crystal at different temperatures as a function of frequency.

The dielectric constant is calculated using the formula

$$
\varepsilon_{r}=\frac{C t}{\varepsilon_{0} A}
$$

where $C$ is the capacitance (farad), $t$ is the thickness (meter), A the area $\left(\mathrm{m}^{2}\right), \varepsilon_{0}$ is the absolute permittivity of the free space having a value of $8.854 \times 10^{-12} \mathrm{Fm}^{-1}$.

It is seen that the value of a dielectric constant is found to decrease and attain constant values in the higher frequency region. The decrease in the dielectric constant of the title crystal at low frequencies may be attributed to the contribution of the electronic, ionic, orientation, and space charge polarizations which depend on frequencies. At low frequencies all the four contributions are active [9]. The low values of $\varepsilon_{\mathrm{r}}$ at high frequencies are important for these materials in the construction of photonic and NLO devices, which suggests that the sample possesses enhanced optical quality with low level defects [10]. Dielectric loss decreases with an increase in frequency. A larger value of $\varepsilon_{\mathrm{r}}$ and $\tan \delta$ at low frequency arises, due to the presence of space charge polarization near the grain boundary interfaces which depends on the purity and perfection of the sample.

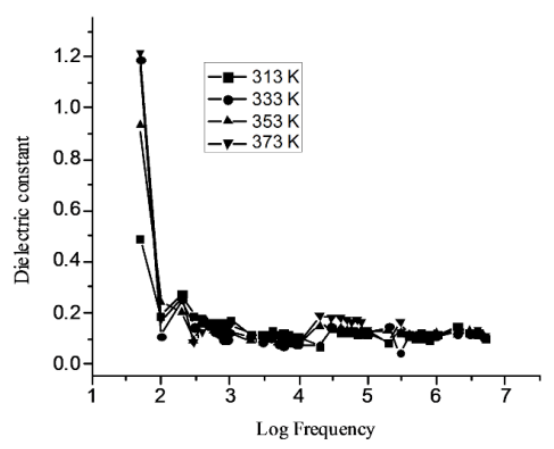

Fig. 4. Variation of dielectric constant with frequency for different temperatures of LCSNC crystal. 


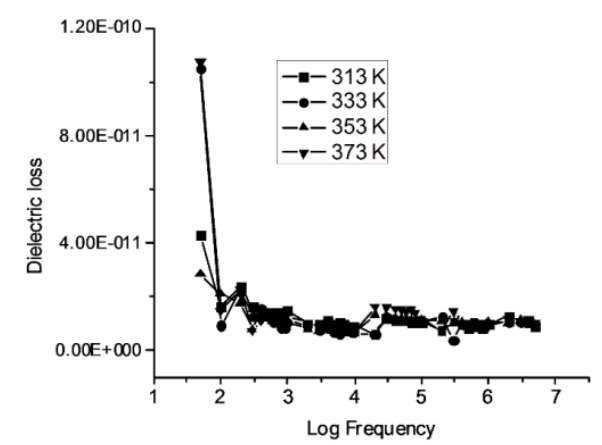

Fig. 5. Variation of dielectric loss with frequency for different temperatures of LCSNC crystal.

The second harmonic generation behavior of the powdered material was tested with the Kurtz and Perry method [11] using a Q-switched $\mathrm{Nd}$ : YAG laser $(\lambda=1064 \mathrm{~nm})$ with a pulse duration of $10 \mathrm{~ns}$. An intense green light was observed and this confirms that the grown crystals are SHG active. The amplitude of the SHG output voltage was measured using a photomultiplier and a digitalizing oscilloscope assembly. The SHG efficiency was found to be about 0.51 times that of the standard KDP crystal. Hence, it is used for applications in photonic and optoelectronic devices.

In conclusion, single crystals of LCSNC were grown by a slow evaporation technique. Single crystal XRD confirmed the unit cell parameters. The UV-Vis study reveals the suitability of the crystal for NLO applications and the lower cutoff wavelength is found to be $203 \mathrm{~nm}$ and the upper cutoff wavelength is $328 \mathrm{~nm}$. There is no absorption of wavelength in the entire visible region. The FTIR spectrum gives various functional groups present in the structure. From the dielectric study, it is found that both dielectric constant and dielectric loss of the crystal decrease with an increase in frequency. The emission of a green signal confirms the second harmonic generation efficiency of the crystal. Thus, the moderate SHG efficiency and encouraging dielectric properties of the crystal indicate the suitability of this crystal for photonic device fabrication.

\section{References}

[1] D.S. Chemla, J. Zyss, Nonlinear optical properties of organic molecules and crystals (Academic Press 1987).

[2] P.N. Prasad, D.J. Williams, Introduction to Non Linear Optical Effect in Molecules and Polymers (John Wiley \& Sons, New York, 1991).

[3] M. Loganayaki, P. Murugakoothan, Asian J. Chem. 23, 5085 (2011).

[4] G. Bhagavannarayana, S. Kumar, M. Shakir, S.K. Kushawaha, K.K. Maurya, R. Malhotra, K.R. Rao, J. Appl. Cryst. 43, 710 (2010).

[5] J. Packiam Julius, A. Joseph Arul Pragasam, S.A. Rajasekar, S. Selva Kumar, A. Stephen, P. Sagayaraj, J. Cryst. Growth 267, 619 (2004).

[6] W. Kemp, Organic Spectroscopy (Palgrave Macmillan, 1991).

[7] S. Zulifiqar Ali Ahamed, G.R. Dillip, L. Manoj, P. Raghavaiah, B. Deva Prasad Raju, Phot. Lett. Poland 2(4), 183 (2010).

[8] S. Boomadevi, H.P.Mittal, R. Dhanasekaran, J. Cryst. Growth 261, 55 (2004).

[9] K.V. Rao, A. Smakula, J. Appl. Phys. 36(6), 2031 (1965).

[10] B.D. Halton, K. Landskron, W.J. Hunks, Materials Today 9, 22 (2003).

[11] S.K. Kurtz, T.T. Perry, J. Appl. Phys. 39, 3798 (1968). 